\title{
Frontières
}

\section{Le cadavre sacré}

\section{Le cas du Soldat Inconnu}

\section{Claude Javeau}

Volume 19, numéro 1, automne 2006

Enjeux politiques et mort

URI : https://id.erudit.org/iderudit/016631ar

DOI : https://doi.org/10.7202/016631ar

Aller au sommaire du numéro

\section{Éditeur(s)}

Université du Québec à Montréal

ISSN

1180-3479 (imprimé)

1916-0976 (numérique)

Découvrir la revue

Citer cet article

Javeau, C. (2006). Le cadavre sacré : le cas du Soldat Inconnu. Frontières, 19(1), 21-24. https://doi.org/10.7202/016631ar

\section{Résumé de l'article}

Peu après la fin de la Grande Guerre, divers États vainqueurs ont inhumé à une place d'honneur un soldat inidentifiable, qu'on a appelé Inconnu. Celui-ci devenait « le Fils de toutes les mères qui n’ont pas retrouvé leur Fils », le métonyme de tous les soldats tombés pour la Patrie, dont leurs mères était la métaphore. Les rites qui sont liés à la présence de ce corps méconnaissable présentent les divers aspects des rites funéraires en général, mais portés au niveau de cérémonies commémoratives, dont la signification se perd, qui sont à la fois d'expiation (de toutes ces morts glorieuses) et d'exaltation de la communauté nationale.
Ce document est protégé par la loi sur le droit d'auteur. L'utilisation des services d'Érudit (y compris la reproduction) est assujettie à sa politique d'utilisation que vous pouvez consulter en ligne.

https://apropos.erudit.org/fr/usagers/politique-dutilisation/ 


\section{Résumé}

Peu après la fin de la Grande Guerre, divers États vainqueurs ont inhumé à une place d'honneur un soldat inidentifiable, qu'on a appelé Inconnu. Celui-ci devenait "le Fils de toutes les mères qui n'ont pas retrouvé leur Fils», le métonyme de tous les soldats tombés pour la Patrie, dont leurs mères était la métaphore. Les rites qui sont liés à la présence de ce corps méconnaissable présentent les divers aspects des rites funéraires en général, mais portés au niveau de cérémonies commémoratives, dont la signification se perd, qui sont à la fois d'expiation (de toutes ces morts glorieuses) et d'exaltation de la communauté nationale.

Mots clés: Grande Guerre - corps méconnaissable - rites funéraires.

\section{Abstract}

In the aftermath of the Great War, several nations buried an unidentified Soldier in commemorative tombs, which are hitherto known as the Tomb of the Unknown Soldier. He becomes "the Son of Mothers who could not find their Son", a metonym for all the fallen soldiers who gave their life to protect their Fatherland, thereby transforming mothers into a powerful living metaphor. The rites used to evoke the presence of this unidentified corpse bear various aspects of funeral rites in general, which are elevated to the status of a commemorative ceremony, at once atoning for all these "glorious dead" and exalting national community - a commemorative ceremony whose signification is waning.

Keywords: Great War- unidentified corpse - funeral rites.

\section{LE CADAVRE SACRÉ LE CAS DU SOLDAT INCONNU}

\begin{abstract}
Claude Javeau,
professeur émérite de sociologie, Université libre de Bruxelles.
\end{abstract}

Le 11 novembre 1919, un an jour pour jour après la mise en œuvre du cessezle-feu sur le front occidental de la Première Guerre mondiale, que marque la conclusion de l'Armistice, un cercueil drapé des couleurs de la France est amené sur l'affût d'un obusier de 155 au pied de l'Arc de triomphe de l'Étoile à Paris, où on l'enterre. Il contient celui qu'on appellera le Soldat inconnu, sur la pierre tombale duquel on installera un brûleur destiné à alimenter une flamme qui ne devra cesser de brûler et soumise à des "ravivages» à l'occasion de cérémonies solennelles (le 14 juillet, jour de la fête nationale, le 11 novembre, jour de la commémoration de l'armistice de 1918). Le corps qui a été ainsi translaté vers cet endroit hautement symbolique qu'est l'Arc de triomphe (le triomphe en question étant celui des armées de la République et de l'Empire contre les ennemis coalisés de la France) a été désigné parmi d'autres corps mis en bière par un soldat tiré au hasard. La scène a été représentée par Bertrand Tavernier dans son film La vie et rien d'autre de 1988: on y voit Philippe Noiret, qui campe un médecin major chargé d'identifier des corps de soldats ensevelis dans un tunnel ferroviaire où leur train a explosé, y tenir des propos grinçants au général qui préside la cérémonie. Rappelons que dans ce très beau film, Sabine Azéma joue le rôle d'une veuve issue de la bonne bourgeoisie, venue à la recherche de son mari tué, et qui fait la connaissance de la maîtresse de celuici, d'extraction beaucoup plus modeste.

Si Soldat inconnu il y a, c'est que son corps, non réclamé, était méconnaissable ou du moins non identifiable. L'histoire ne dit rien de l'état du cadavre. La qualification d'inconnu ne correspond pas à celle de méconnu. On parlera souvent de l' "Inconnu », tout court, sans autre précision, son rapport à la Grande Guerre semblant aller de soi pour les générations à venir. D'autres pays imiteront la France, entre autres la Grande-Bretagne, où il est inhumé à l'abbaye de Westminster; l'Italie, où sa tombe a été placée sous l'Autel de la Patrie, situé au sein du grand monument de l'Italie unifiée, le Vittoriano; en Belgique, où il fut déposé au pied de la Colonne du Congrès, symbole de l'indépendance du pays. Citons encore la Grèce et les ÉtatsUnis. Ces tombes ont bien pour caractère spécifique d'abriter les restes d'un individu auquel on n'a pu donner un nom.

Que l'inconnu vienne à être identifié,

il faudrait le remplacer. Par leur caractère de reconnaissance individuelle audelà de l'impuissance à nommer, ces tombeaux sont également des monuments funéraires commémoratifs. Plus que des ossuaires, les tombeaux des soldats inconnus ont servi de support à la ritualisation du deuil par le biais des grandes commémorations nationales. (Capdevilla et Voldman, 2002, p. 231.) 
Lieu de mémoire insigne, la tombe de l'Inconnu français devait répondre à une préoccupation que rappelle le général Weygand treize ans après son inauguration :

Donner une tombe insigne à un soldat inconnu qui serait le «Fils de toutes les Mères qui n'ont pas retrouvé leur Fils »[...] donner à ces familles [des disparus] une tombe où elles pourraient prier [...] Cette idée d'honorer les plus modestes et les plus obscurs de nos héros faisant porter l'hommage sur un soldat inconnu répondait à un sentiment universel. (Capdevilla et Voldman, 2002, p. 231)

Notons que les familles des disparus n'ont jamais pris le pli de venir prier sur le tombeau de l'Inconnu, qui constitue un lieu frappé d'interdits officiels. Seules des cérémonies officielles l'ont pris pour point de ralliement. Les hommages privés ne sont pas de mise. Les «plus modestes et les plus obscurs» ne sont point souhaités à l'Arc de triomphe. Les puissants y ont accès, dans la mesure où ils représentent un corps social, national ou étranger. Des associations patriotiques sont également bienvenues, pour des hommages d'anciens à celui qui pourrait être un des leurs et qui, en fait, symbolise tous les «leurs» possibles. Il en va de même, en général, dans les autres pays. À Londres, où le cénotaphe érigé dans l'attente de la translation des restes du Soldat inconnu à Westminster a acquis un droit à une existence en principe perpétuelle, c'est autour de ce monument que se célèbrent les cérémonies commémoratives de la proclamation de l'armistice, le dimanche le plus proche du 11 novembre. Dressé à Whitehall, il reçoit les dévotions patriotiques de personnalités les plus hautes du royaume, reine en tête, ainsi que des représentants de tous les pays membres du Commonwealth. L'évêque (anglican) de Londres y prononce un bref discours dans lequel se trouve exalté le sacrifice de tous les morts de toutes les guerres $\mathrm{du} \mathrm{XX}^{\mathrm{e}}$ siècle. La cérémonie prend donc une allure politico-religieuse fort typique des usages britanniques, et est entièrement transmise en direct sur les antennes de la télévision publique.

\section{FILS DE TOUTES LES MÈRES QUI N'ONT PAS RETROUVÉ LEUR FILS}

Je reviens à ce passage de la citation du général Weygand. Ce qui frappe d'emblée est que seules les mères sont invoquées (qu'on ne parle que de fils est normal dans la mesure où à cette époque très peu de femmes, outre des infirmières, étaient mobilisées dans les armées, et surtout œuvraient sur le front). Pourtant les soldats morts ont aussi des pères, et il n'est pas permis de supposer que les pères n'ont pas aussi et aussi sincèrement que les mères pleuré leurs fils morts à la guerre. Du reste, ils peuvent aussi être eux-mêmes des pères, et encore des époux, des fiancés, des amants. Mais il est typique d'un certain discours de ce temps-là sur la douleur d'affecter celle-ci aux seules femmes. La figure sollicitée ici est celle de la mater dolorosa. Celle-ci vient se ranger non seulement aux côtés de la mère patrie, mais également de Marianne, figure féminine qui symbolise la République. On citera par surcroît une autre figure emblématique, celle de la Madelon, héroïne d'une chanson composée pour les troupiers au début de la guerre, qui propose de celle-ci une image "fraîche et joyeuse », contrastant avec celle qui prévaut dans les chants nés réellement au sein de la troupe, à l'exemple de la célèbre et subversive Chanson de Craonne. C'est toutefois ici l'image de la mère qui a été retenue. À l'instar de la Vierge Marie telle qu'elle est représentée dans les Pietà, avec le corps de son divin Fils mort étendu sur ses genoux, les mères des fils tués à la guerre versent sur leur corps toutes les larmes de leurs propres corps. Mais il y a une différence, et elle est de taille: les corps des fils n'ont pas été retrouvés ou, du moins, n'ont pu être identifiés. Cette absence renforce considérablement la douleur des mères. La présence du corps de l'Inconnu, sous sa flamme à l'Arc de triomphe ou au pied de la Colonne du Congrès, est censée compenser toutes ces absences. Ce corps est à ce point magnifié, symboliquement "grandi », qu'il peut prétendre figurer tous les corps manquants sous les yeux des mères, ceux dont seuls les noms, et encore seulement lorsque leur mort est avérée, témoignent, par leur inscription sur les monuments aux morts, de leur existence passée. Restent tous les disparus dont la mort n'a jamais été prouvée, et au sujet desquels les imaginations peuvent vagabonder.

Il est permis, me semble-t-il, d'avancer que «les mères » sont ici la métaphore de la patrie (on notera ce curieux changement de genre, sauf pour ce qui est du genre grammatical: si Patrie est bien du genre féminin, elle évoque, par son étymologie, le père et c'est donc une figure paternelle que les mères en question métaphorisent, mais il est vrai aussi que l'on parlera volontiers de mère patrie). C'est la France, en l'occurrence (mais cela peut aussi valoir pour la Belgique, la Grande-Bretagne, l'Italie, etc.), qui n'a pas retrouvé tous ses fils, c'est-àdire la totalité des soldats morts, membres d'une armée qui, pour avoir disparu, n'en est pas moins, à l'instar de l'Église qualifiée de même manière, triomphante. Triomphante, car ses membres ont été jusqu'au sacrifice suprême. Tous les soldats tués sont nécessairement glorieux même si leur décès est survenu à l'arrière du front à la suite d'une maladie. Pourvu que celle-ci ait été contractée au front et résulte de leur présence au front, la gloire ne sera pas marchandée à ces défunts-là aussi. Ce sacrifice suprême, ils l'ont accompli pour que vive la Mère France, cette patrie à qui chaque fils, autant qu'à chaque mère biologique, et sans doute davantage en temps de guerre, doit la vie (dans le célèbre duo de La Muette de Portici, l'un des chanteurs ne clame-t-il pas «à mon pays je dois la vie» ?). À la fin de la guerre, la Patrie faisant le compte des soldats qui ont donné, certes souvent nolens volens, mais l'héroïsme volontaire leur est généreusement reconnu, leur vie pour elle, découvre qu'il en est beaucoup sur lesquels elle ne peut mettre un nom. Ils sont condamnés à rester inconnus. L'Inconnu par excellence est censé les représenter tous, être en quelque sorte leur porte-parole à l'égard des citoyens en général, et aussi des soldats survivants, qui doivent trouver en lui un « interlocuteur valable » lors des cérémonies patriotiques.

Il convient de remarquer que des tombeaux de soldats inconnus n'ont été instaurés que dans les pays figurant du côté des vainqueurs de la Grande Guerre, à l'exception de la Turquie, comme l'indique la citation ci-dessous.

Une tentative d'ériger un mémorial au Soldat inconnu en Allemagne, en 1923, engendra des combats de rue entre groupes favorables et défavorables à la guerre. L'amertume de la défaite avait affecté trop sensiblement les survivants pour qu'ils s'accordent sur une commémoration appropriée. La mémorialisation la plus importante devint le monument de Tannenberg, lieu de la défense héroïque de la PrusseOrientale contre les Russes en 1914. Il fut choisi pour sépulture du président Hindenburg, vainqueur de Tannenberg, en 1934. Déplacé en raison de l'avancée de l'Armée rouge en 1945, il est à présent situé à Hambourg. En Turquie, l'un des plus poignants des monuments commémoratifs de la guerre fut érigé par Kemal Atatürk à Gallipoli, où il avait commandé les troupes qui défendaient en 1915 la péninsule contre les Britanniques, les Australiens, les NéoZélandais, les Indiens et les Français. L'inscription qu'il porte prie généreusement toutes les personnes endeuillées d'honorer ceux qui moururent égaux dans le sacrifice. (Keegan, 2002,

p. 405. Ma traduction.)

Le nombre de soldats non identifiés n'est pas d'ordre marginal. Pour prendre un seul exemple, dans le cimetière pour soldats allemands de la Grande Guerre de Belleau, dans l'Aisne, on compte 4307 corps identifiés pour 4322 corps non identifiés 


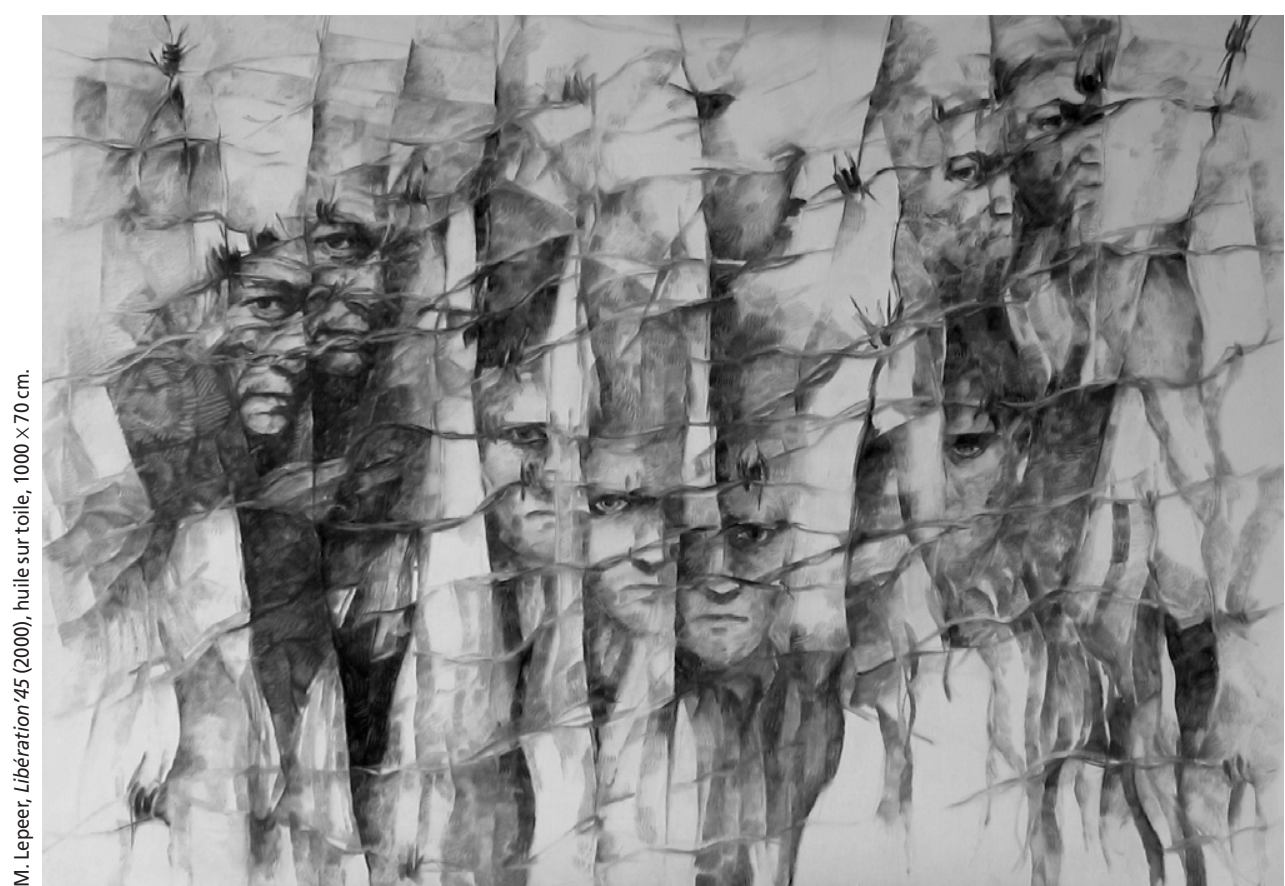

(Garfield, 1990, p. 27). L'Inconnu ne représente pas une poignée d'inconnus. À côté de ces corps non identifiés, d'ailleurs, on doit encore compter tous ceux dont il n'est resté aucune trace, brûlés dans des incendies ou volatilisés dans un bombardement. Le nombre de mères n'ayant pas retrouvé leurs fils est très élevé, et la patrie n'est pas loin de constituer, pour celles-ci, une immense nécropole d'anonymes. Paradoxalement, l'Inconnu donne un nom à ces anonymes. C'est un des leurs, dont les noms oubliés s'hypostasient en celui qu'il porte pour les générations à venir. Inconnu, c'est aussi un nom. Il est connu sous ce nom. Remarquons qu'on ne l'a pas appelé l'Anonyme. Inconnu implique qu'il aurait pu être connu. Et, comme rappelé plus haut, s'il arrivait qu'il fût identifié, on devrait le remplacer par un autre, réputé non identifiable au moment de ce remplacement. L'Inconnu a porté un nom jusqu'au moment où il a rencontré la mort, sous l'une des formes que celleci revêt dans les circonstances guerrières, balle ou baïonnette, gaz ou microbe, obus ou bombe aérienne.

Si le collectif «les mères » se métaphorise en patrie, l'Inconnu, lui, est la métonymie de tous les soldats morts à la guerre et à cause d'elle (en sont exclus, évidemment, ceux qui ont été exécutés pour désertion, insubordination ou simplement «pour l'exemple»). On pourrait en dire autant de Hindenburg, censé incarner les vertus du guerrier allemand. Mais l'identification est ici moins aisée que dans le cas de l'Inconnu français, belge, italien, etc. Hindenburg est un grand chef, prestigieux vainqueur d'une bataille justement célébrée (en réalité, le véritable artisan de cette victoire a été son chef d'état-major, Ludendorff). Il est difficile pour le troupier ordinaire de se reconnaître dans cet imposant héros. Tandis qu'il peut le faire dans l'Inconnu, dont le grade et la fonction sont aussi inconnus que son patronyme, et qui n'est un héros que par est avant tout celui du soldat ordinaire, qui n'est pas nécessairement l'auteur d'une action d'éclat. Et l'eût-il été, que l'on aurait perdu le souvenir de celle-ci, comme on a perdu celui du nom de son auteur.

\section{L'INCONNU MÉCONNAISSABLE EST UN CORPS EXPIATOIRE}

Comme tous les défunts, le Soldat inconnu a été, lors de son inhumation, l'objet d'une ritualisation spécifique répondant à la qualification de «piaculaire» introduite par Durkheim:

Mais il existe aussi des fêtes tristes qui ont pour objet ou de faire face à une calamité ou, tout simplement, de la rappeler ou de la déplorer [...] Nous proposons d'appeler piaculaires les cérémonies de ce genre. Le terme de piaculum a, en effet, cet avantage que, tout en éveillant l'idée d'expiation, il a pourtant une signification beaucoup plus étendue. Tout malheur, tout ce qui est de mauvais augure, tout ce qui inspire des sentiments d'angoisse ou de crainte nécessite un piaculum et, par conséquent, est appelé piaculaire. Le mot paraît donc très approprié à désigner des rites qui se célèbrent dans l'inquiétude ou dans la tristesse. [...] Le deuil nous offre un premier et important exemple de rites piaculaires. (Durkheim, 1985, p. 55-56) pétition de principe. Son héroïsme présumé
La translation des restes de celui qui avait été choisi pour être l'Inconnu a, sans conteste, constitué une «fête triste» (le mot «fête», utilisé par Durkheim, semble ici peu approprié). Ces restes ont fait l'objet de rites préliminaires, pour reprendre la terminologie de Van Gennep. Il s'est agi, comme à tout défunt, de lui dire adieu. Le «sentiment universel» de peur de la contagion de la mort par le mort, décrit par Lévy-Bruhl, est domestiqué par l'intérêt accordé au cadavre, lors de diverses pratiques de purification et d'hommage qui sont autant de rites d'oblation. En l'occurrence, ces rites ont revêtu les espèces d'une cérémonie patriotique célébrée en grande pompe. Garde d'honneur, drapeaux, prises d'armes, uniformes chamarrés, discours enflammés, musiques guerrières et funèbres, hymne national, défilé d'enfants des écoles, représentants des corps constitués en habits de deuil, etc. Ces rites sont répétés en moindre envergure à chaque cérémonie du rallumage de la flamme, symbole de l'éternité glorieuse offerte à l'Inconnu, en même temps que témoin des adieux qui lui ont été faits et qui lui sont réitérés.

Les rites préliminaires de séparation devraient être suivis de rites liminaires, de la marge, qui se rapportent au seuil entre le monde ancien, celui où les morts étaient encore vivants, et le monde nouveau où ils sont devenus morts. Ils soulignent l'entrée dans la période de deuil. En l'occurrence, cette période avait déjà débuté, pour chaque famille concernée, dès l'annonce de la disparition du fils tombé «face à l'ennemi ». La translation des restes de l'Inconnu combine ces deux étapes rituelles : la pompe militaire, grandiose dans sa mise en scène, a été suivie de l'inhumation en lieu fortement porteur de signification civique. Il s'agit bien là d'une attention particulière à l'égard de ceux et de celles - surtout de celles! - qui doivent porter le deuil de tous ceux que l'Inconnu synthétise et symbolise. L'honneur insigne qui lui est fait d'une sépulture, dont on ne pourrait trouver de plus digne, se répand ainsi sur les endeuillé(e)s qui sont censés ne pouvoir espérer mieux pour leur commun fils, donc pour tous les fils concrets qu'ils et elles pleurent. La marge, qui avait été franchie il y avait parfois pas mal de temps déjà, fait l'objet d'une répétition. Elle quitte le domaine privé pour le domaine public, mais elle n'ouvre pas la période, elle ne fait que la réaffirmer en lui conférant un décorum inhabituel.

Les rites d'intégration ou d'accueil, dits postliminaires, soulignent la réception du mort dans la société des disparus. Il s'agit en fait de la réintégration posthume de ces soldats défunts, privés de fonction par la mort, dans la collectivité nationale, qui leur attribue une nouvelle fonction sociale, celle de 
héros, qui est aussi celle de martyr. Discours officiels, drapeaux, hymnes patriotiques ponctuent ces célébrations qui se répètent sous des formes généralement simplifiées lors de chaque cérémonie commémorative. L'Inconnu rejoint les morts illustres; dans une certaine mesure, à Paris du moins, sa tombe est une succursale du Panthéon. Mais là où on a inhumé les grands hommes à qui la patrie est reconnaissante, tous les disparus ont un nom censé être destiné à être à tout jamais célèbre; tandis que l'Inconnu, lui, représente une multitude d'anonymes. Son nom d'Inconnu ne peut être célèbre à la façon de ceux de Victor Hugo, de Jean Jaurès ou de Jean Moulin. Mais sa gloire, dans une certaine mesure, est plus grande que la leur. Elle est celle de la patrie tout entière.

Si l'on retient l'aspect «piaculaire » de ces rites, on peut avancer l'idée qu'ils manifestent aussi des sentiments de repentir, sinon de véritable expiation, à l'égard de tous ces jeunes gens que la mère patrie, certes forcée par les événements (mais il est entendu qu'on ne fait jamais la guerre que parce qu'on y est contraint: même Hitler, pour envahir la Pologne le $1^{\mathrm{er}}$ septembre 1939, a usé d'un subterfuge de prétendue attaque surprise des Polonais qui devait justifier une fallacieuse « riposte »), a envoyés à la mort. Au-delà de l'expiation, toutefois, se donne à voir une immense tristesse, celle que condense en un seul torrent toutes les larmes présumées des mères dont les fils tués n'ont pu être identifiés. La mort héroïque des guerriers fabrique des morts méconnaissables, dont la vue est insupportable. Car c'est bien de corps qu'il s'agit, de cadavres, de ces objets qui, nous dit Origène, n'ont «de nom dans aucune langue», a fortiori quand il n'est plus possible, vu leur état physique, de leur donner le nom des vivants qu'ils avaient été.

Le Soldat inconnu est avant tout un corps. Sous la dalle sacrée, c'est un objet concret, un cadavre, qui a été inhumé. On aurait pu se contenter d'une représentation visuelle en deux ou trois dimensions, à l'instar des crucifixions peintes ou sculptées que l'on trouve dans les églises catholiques. Mais on sait que celles-ci abritent souvent des reliques qui peuvent être quelquefois des cadavres entiers. Ces corps sont ceux de bienheureux et de saints, qui jouent ainsi le rôle de suppléants de celui du Christ. Rappelons que l'existence de reliques dans des religions séculières, dont le marxismeléninisme et ses dérivés sont l'illustration la plus immédiatement présente à l'esprit, constitue le but de pèlerinages soigneusement organisés. Si le corps embaumé de Mao, à Pékin, hypostasie en principe un régime qui se réclame encore de lui, à Moscou, la momie de Lénine continue à trôner à la place Rouge alors que le régime qu'il avait créé s'est écroulé. Pendant trois ans, cette momie a été doublée par celle de Staline qui fut retirée du mausolée à la suite des révélations de Khrouchtchev au $\mathrm{XX}^{\mathrm{e}}$ Congrès du PCUS, et transférée dans une sépulture ordinaire, creusée à côté de celles d'autres dignitaires du Parti, derrière le mur d'enceinte du Kremlin. Cette translation a signifié alors la punition infligée à un homme dont les crimes avaient enfin été officiellement reconnus. On peut imaginer qu'un sort semblable serait réservé à l'Inconnu s'il était inopinément prouvé que son corps était celui d'un déserteur. Et d'ailleurs, comme il a été rapporté plus haut, le simple fait de pouvoir lui donner un nom entraînerait son éviction de l'emplacement d'honneur qui lui avait été réservé en exclusivité.

\section{LE SOLDAT INCONNU}

\section{EST AVANT TOUT UN CORPS.}

\section{SOUS LA DALLE SACRÉE,}

\section{C'EST UN OBJET CONCRET,}

\section{UN CADAVRE, QUI A ÉTÉ INHUMÉ.}

Le rapport de la sanctification civile de l'Inconnu avec la pratique chrétienne d'inhumation en terre consacrée est clairement dicible. L'Inconnu est le martyr par excellence d'une nation elle-même martyrisée par la guerre. (Ce qui, s'agissant des ÉtatsUnis, ne peut résulter que d'une espèce de contamination, le pays n'ayant pas été envahi et sa population n'ayant guère eu à souffrir de l'état de guerre.) Son corps constitue donc une relique au sens chrétien du terme. À chaque cérémonie patriotique qui se déroule autour de son tombeau se renouvelle l'hommage à cette relique, support d'un culte civique qui emprunte beaucoup au culte des morts dans la tradition chrétienne (catholique dans les pays où cette confession était largement majoritaire à l'époque de l' «invention» des Inconnus). Quoi qu'on puisse penser du cachet morbide qui affecte ce culte dans la plupart de ses manifestations, il convient de reconnaître que celles-ci ne peuvent se passer de la présence concrète des corps des défunts. Et si le monument aux morts, dans les communes, n'a pas été bâti sur des tombeaux, quand il n'est pas érigé au cimetière local où se trouvent les tombes des citoyens morts au combat, il est dressé à proximité d'une église, lieu de conservation du souvenir de tous les morts du pays.

On a pu, du reste, souligner combien la pratique de la crémation dont on pouvait penser qu'elle débouchait sur une dispari- tion complète des corps incinérés à la suite de la dispersion de leurs cendres, a connu, depuis quelque temps, une re-ritualisation dont les marques de déférence adressées à ces cendres sont l'une des composantes majeures :

Comme j'ai essayé de le montrer, la pratique, en voie d'extension constante, de la crémation ne fait pas, contrairement à des attentes que l'on pourrait qualifier en l'occurrence d'hyper-matérialistes, l'économie d'une ritualisation (en l'occurrence, dite « cinéraire») qui, pour être par certains aspects spécifiques, se rattache aux modes de ritualisation des obsèques et $\mathrm{du}$ deuil en vigueur sous nos latitudes.

(Javeau, 2003, p. 75)

Peut-être est-il permis de supposer que si l'Inconnu avait dû être institué à notre époque, c'est sous les espèces de cendres que sa dépouille aurait été proposée au culte patriotique. S'agissant de ce dernier, il semble évident que ses significations s'estompent sensiblement au sein des populations contemporaines. Le corps de l'Inconnu n'est plus que le support éloigné d'une mémoire elle-même ritualisée. Les gardiens de cette mémoire ne sont plus que les ordonnateurs de pompes funèbres officielles qui n'attirent plus guère de participants et de spectateurs. Cependant, le corps de l'Inconnu est toujours présent et il n'est pas dépossédé du mana dont est porteur tout cadavre, même réduit à l'état de cendres s'il échet. Que ce mana puisse être lové dans les plis du drapeau national n'apparaît sans doute plus clairement aux yeux de la majorité des citoyens dont l'Inconnu était censé représenter la fraction glorieuse. Mais la sépulture reste un lieu de rassemblement officiel et, pour certains, un lieu de réaffirmation d'unité nationale, comme c'est le cas en Belgique, État menacé d'implosion. Si l'exaltation patriotique qui a accompagné la mise au tombeau de l'Inconnu est devenue à tout le moins objet de perplexité, ce tombeau lui-même reste, pour quelque temps encore, un lieu de mémoire.

\section{Bibliographie}

CAPDEVILLA, L. et VOLDMAN, D. (2002). Nos Morts. Les sociétés occidentales face aux tués de la guerre, Paris, Payot.

DURKHEIM, E. (1985). Les formes élémentaires de la vie religieuse, Paris, PUF, coll. Quadrige.

GARFIELD, J. (1990). The Fallen, Londres, Leo Cooper.

JAVEAU, C. (2003). «Retour sur les rites piaculaires: pratiques et rôles dans l'immédiat et à distance», Études sur la Mort, $n^{\circ} 123$, p. 69-78.

KEEGAN, J. (2002). The First World War. An Illustrated History, Londres, Pimlico. 